\title{
Color Texture Description with Diagonal Local Binary Patterns Using a New Distance Metric for Impressive Image Retrieval
}

\author{
Vijaylakshmi Sajwan \\ ${ }^{1}$ Research Scholar, Dept. of Computer Science \& Engg. \\ Himgiri Zee University, Dehradun, Uttarakhand, India \\ vijaylakshmi@live.com \\ Dr. Rakesh Ranjan \\ ${ }^{2}$ Professor, Dept. of Engineering, Himgiri Zee University \\ Dehradun, Uttarakhand, India \\ rakesh.ranjan@hzu.edu.in \\ Sajal Swapnil \\ 3Scholar, Dept. of Electronics and Communication Engineering, NIT \\ Kurukshetra, Hariyana, India \\ Sajalswap09@gmail.com
}

\begin{abstract}
With the reliability and dependence of the world over the digitalization of information in all spectrums of professional and personal life, the volume of digital data is increasing exponentially. With such a huge database, retrieving information, and images from the storage media pose serious challenges. In this paper, a diagonal local binary pattern for color images (DLBPC) and a new distance metric are proposed for the retrieval of data with high accuracy and speed. The descriptor employs the deviceindependent $L * a * b *$ color space. The discriminative quality of DLBPC is enhanced by combining local features with the (color moment) global feature of the image for effective image retrieval. To assess the efficacy, the suggested system was implemented on challenging datasets: Wang $-1 \mathrm{~K}$ and GHIM-10K. The analysis findings demonstrate the efficacy of the suggested system over other reported systems.
\end{abstract}

Keywords: Diagonal local binary pattern color image (DLBPC), Local feature, global feature, LBP, Color moment, new distance metric.

\section{Introduction}

CBIR is a tool for locating an image from a large archive formulated on the user's requirements. Query by image content is another name for CBIR (QBIC). CBIR ([1], [2], [3], [4], [5]) is a feasible option to standard TBIR, which has many disadvantages. Picture segmentation is covered by CBIR, which removes an attribute from the image and transforms it into a semantic feature. "We extricate low-level (color, texture, and shape) or high-level (machine learning technique) features from images in general" [6]. Regardless of 30 years of exploration, quite possibly the most elaborate examination field in the field of AI and pattern recognition is CBIR. Many researchers have been drawn to devise an approach that provides high retrieval rates in a short amount of time as a result of the search for more and easier CBIR systems. An image retrieval system teaches a user how to easily navigate, search, and retrieve images from a database. Data security, digital libraries, biodiversity, medical imaging, crime prevention, video surveillance, and human-computer interaction is just a few of the applications for these databases [7].

The CBIR frameworks are heavily reliant on two processes: feature extraction and have matched. The most vital component is feature extraction, which permits an image to be represented by a strongly appropriate feature that varies only slightly within intra-class and inter-class images. In general, characteristics representation is divided in two ways: (1) global and (2) local.

A global method derives attributes from the entire image while disregarding local properties and structural relationships. Both are effective in terms of processing and immune to image noise. Many of the issues associated with occlusion, views and lighting changes, and native features of image shapes are insufficiently addressed by global techniques. This type of matter is successfully handled by local feature extraction technologies, which draw out an attribute from block areas of an image. 
Tamura texture contents [9], Gabor filters [10], gray level co-occurrence matrices [11], and the Markov random field model [12] are all texture-based global content extraction processes. SIFT [13], LBP [14], SURF [15], Daisy [16], PCA-SIFT [17], HOG [18], BRIEF [19], WLD [20], Rank-SIFT [21], and several others are successful local feature extraction methods. Color and texture provide essential details for evaluating efficient functionality and retaining retrieval systems' high performance. The grayscale images were given the traditional texture features. "Within the state-of-the-art identification and classification scheme, SIFT has proven to be the four most effective and reliable local texture descriptors for grayscale images" [7]. LBP is a significant descriptor that represents local texture characteristics that are untouched by variations in lighting. The LBP operator has been used to classify textures [25, 27], recognize faces [29-31], recognize facial expressions [32, 33], and retrieve images [34-37], among other things. The bulk of research on the classic LBP descriptor and its variants have been performed on grayscale images. The growing popularity of the LBP operator in a wide range of realistic applications have prompted researchers to build descriptors that can precisely reflect color texture patterns in the same way that the LBP operator does with grayscale pictures. "As a grayscale image, the grayscale LBP operator naturally applies to process each channel of a color picture" [1]. This method, known as multispectral LBP (MSLBP), was used to describe color texture in [38]. Later, Maenpaa and Pietikainen [39] experimented with color texture and discovered that three pairs of opponent's color components are adequate to reflect cross-correlation characteristics between the three color channels, rather than six.

We suggest a descriptor named diagonal local binary pattern for color images (DLBPC). This approach depends on converting the RGB color image into $\mathrm{L}^{*} \mathrm{a} * \mathrm{~b} *$ color space and applies a $3 \times 3$ window on the image. Then change the value of the middle pixels in a $3 \times 3$ window depending on the diagonal element of the middle pixel and iterate this method on the unified image. At last for a color image, create an attribute vector using the histogram with 256 bins of a diagonal local binary pattern. To increase the power of the proposed (DLBPC), it was combined with the image's (color moment) global feature to construct a viable image retrieval technique (DLBPC + Color moment). In our second process, we used the $\mathrm{L}^{*} \mathrm{a} \mathrm{b}^{*}$ color space to extract statistical values. These values include: Mean is the first, standard deviation is the second and skewness is the third statistical moment and make a feature vector of each $\mathrm{L}^{*} \mathrm{a} * \mathrm{~b} *$ color channel, it means a total 9 feature will be extracted from the image and then this feature vector combined with diagonal local color texture feature (DLBPC + Color moment).

We introduced a new distance metric in this paper that returns a more identical image in accordance to a test image. The efficiency of all current and proposed trendy methods for image retrieval has been evaluated and compared. The following is a description of the paper's composition. In section 2, we give an overview of some of the latest common local binary pattern-based color picture methods. The suggested descriptors DLBPC and (DLBPC+ color moment) have been derived in section 3. In section 4, we suggested a new distance measure and discussed the image retrieval system's other similarity measures and evaluation measures. Section 5 summarizes the preliminary study of retrieval competence and computing time on numerous color data sets. Section 6 brings the study's findings and recommendations to a close.

\section{Related Work}

Zabih and Woodfill implemented a Census Transform method that is identical to LBP and introduced it at the same time [41]. The census transformation, like the LBP, maps the local vicinity around a pixel in a binary sequence, so the only difference between the two is the order of the bit chain. "LDP calculate higher-order (second, third or more) derivative information along $0^{\circ}, 45^{\circ}, 90^{\circ}$, and $15^{\circ}$ and therefore the attribute vector size for every direction is 59 , so the size of the attribute vector of LDP is $4 \times 59=236$ which contain more detailed and provides a better result than LBP" [6]. The authors suggested Local Tetra Patterns in [44], which encrypt the interaction among the mid pixel and its neighbors. The authors discuss the deterministic the outcome of SVM to forecast query image, as well as using pixel values to erase irrelevant images and reset attribute weights in similarity matching [45].

"The authors of [46] proposed a medical decision support method that excludes features from medical images using GLCM, then PCA to restrict the dimension of a feature vector and classifies the images using SVM to obtain images of tumors and images of multiple sclerosis" [6]. The majority of research on the traditional LBP operator and its variations is focused on grayscale image processing. "Because of the growing demand for color images online and their increasing use for several related applications, the researchers were motivated to create descriptors that could reflect color texture patterns as effectively as the LBP operator does for grayscale images. In their multispectral LBP (MSLBP), they use three channels of a color image and six sets of LBP adversary colors to capture the structural correlation" [38].

Later, "Maenpa and Pieikainen experimented with color and texture and discovered that three pairs of opponent's color components are enough to reflect cross-correlation characteristics among the three color channels, rather than six" [39]. The authors of [48] created LBP histograms for all channels in the YCbCr color space and used PCA to minimize the feature vector's size. Local color vector binary patterns (LCVBPs) for face recognition were developed by the authors in [42]. 
A color histogram was one of several algorithms to help the color information in an image. "Another useful tool is the color correlogram, which takes into account the relationship between different color levels in the structural domain. Despite its benefits, color correlograms are affected by scaling and lighting changes" [52]. The color moment is one among the techniques to extract the color feature from the image using statistical values: mean, standard deviation, and skewness [53].

\section{Proposed Approach}

\subsection{Local Binary Pattern}

"The basic idea behind the local binary pattern is to use the statistical frequency of local structure to precisely define the characteristics of the whole image" [16]. The first LBP operator uses a 3X3 rectangular space with a set dimension. To expose two flaws in the original LBP: fixed scale and minimal texture detail. The authors suggested an updated algorithm based on the concept of the first LBP operator. By applying a fixed scale to a spherical region, the primary flaw is resolved. "Specifically, the radius from the center to its neighbors is not limited to 1 pixel; the number of pixels around the center pixel is not limited to 8 , and all of the operated pixels are not required to correlate to a fixed pixel within the image. By using a circular neighborhood form, (x, y) are the coordinates of the middle pixel $\mathrm{g}_{\mathrm{c}}, \mathrm{R}$ is the radius value of the neighborhood, and $\mathrm{P}$ is the number of sampling points, respectively" [16].

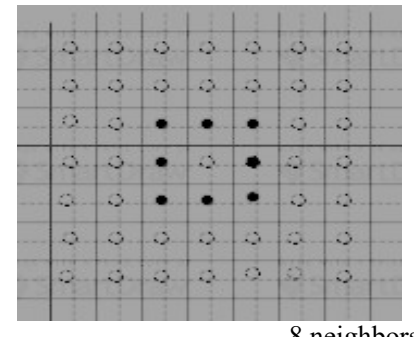

(a)

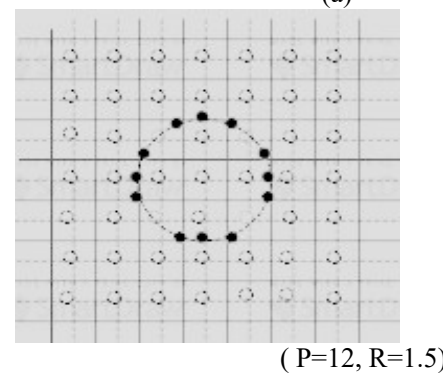

(c)

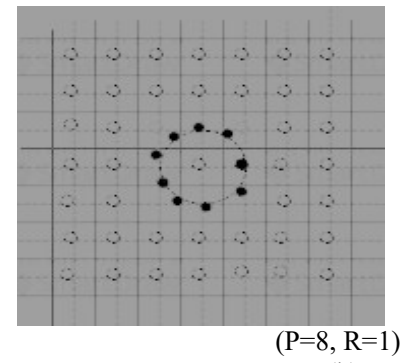

(b)

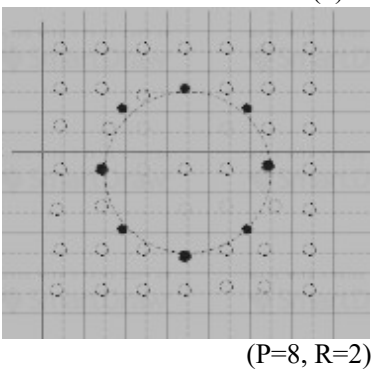

(d)

Fig. 1. LBP operator with different pattern and radius $(P, R)$ : (a) 8 neighbors, (b) $P=8, R=1$, (c) $P=12, R=1.5,(d) P=8, R=2$

The encoding formula is given as: The corresponding LBP for a pixel at (Mc, Nc) can be specified in decimal form as:

$$
\operatorname{LBP}_{\mathrm{P}, \mathrm{R}}(\mathrm{Mc}, \mathrm{Nc})=\sum_{\mathrm{p}=0}^{p-1} s(i p-i c) 2^{p}
$$

After conducting LBP, we can acquire an image known as the LBP continuum, which normally uses a histogram to reflect the spectrum's characteristics. The image's histogram is determined using the following formula:

$$
\begin{gathered}
H=\{h j, j=0,1,2, \ldots \ldots ., n-1\} \\
H j=\sum_{x y} I\{L B P(x, y)=j\}, j=0,1,2, \ldots . . n-1
\end{gathered}
$$

$\mathrm{J}$ is a mode value of LBP, and $\mathrm{n}=2_{\mathrm{p}}$ is the maximum value. $(\mathrm{x}, \mathrm{y})$ is the coordinate of the center pixel. I(.) is the decision function as follows: 


$$
I(m)=\left\{\begin{array}{lll}
1 & \text { if } & m \geq 0 \\
0 & \text { if } & m<0
\end{array}\right.
$$

\subsection{Proposed Diagonal Local Binary Pattern for color image (DLBPC)}

Step 1: Preprocessing: Image preprocessing is included in this step, in which images are transformed into the $* \mathrm{~L} * \mathrm{a} * \mathrm{~b}$ color space, which is intended to describe the human vision. It strives for perceptual uniformity, and its $\mathrm{L}$ aspect closely corresponds to the human sense of lightness.

Step 2: Feature Extraction: This stage is based on the DLBPC operator's avulsion of the image features. The suggested DLBPC operator is predicated on the notion of color pixel partitioning or thresholding using an mdimensional hyperplane. Since $\mathrm{m}=3$ in this particular situation. To generate a thresholding plane in 3-D color space, perform the following steps: Allow a vector $\mathrm{I}(\mathrm{p}, \mathrm{q})=(\mathrm{L}(\mathrm{p}, \mathrm{q}), \mathrm{a}(\mathrm{p}, \mathrm{q}), \mathrm{b}(\mathrm{p}, \mathrm{q}))$ or simply $\mathrm{I}=(\mathrm{L}, \mathrm{a}, \mathrm{b})$ to represent a color pixel within the $\mathrm{L}^{*} \mathrm{a} * \mathrm{~b} *$ color space.

As a result, $\mathrm{m}$ is equal to three color components. Then we add $3 \times 3$ windows to the image and adjust the middle pixel value based on the diagonal element's left and right site neighbors. Repeat this process until you have incorporated the whole image and obtained a DLBPC feature. Finally, build an attribute vector from the histogram of these DLBPC with 256 bins. As seen in Figure 2, we adjust the middle pixel value of the $3 \times 3$ window as follows.

\begin{tabular}{|l|l|l|}
\hline $\mathrm{B}_{1}$ & $\mathrm{~B}_{2}$ & $\mathrm{~B}_{3}$ \\
\hline $\mathrm{B}_{8}$ & $\mathrm{~B}_{0}$ & $\mathrm{~B}_{4}$ \\
\hline $\mathrm{B}_{7}$ & $\mathrm{~B}_{6}$ & $\mathrm{~B}_{5}$ \\
\hline
\end{tabular}$\longrightarrow$\begin{tabular}{|l|l|l|}
\hline $\mathrm{V}_{1}$ & $\mathrm{~V}_{2}$ & $\mathrm{~V}_{3}$ \\
\hline $\mathrm{V}_{8}$ & $\mathrm{~V}_{0}$ & $\mathrm{~V}_{4}$ \\
\hline $\mathrm{V}_{7}$ & $\mathrm{~V}_{6}$ & $\mathrm{~V}_{5}$ \\
\hline
\end{tabular}

Fig. 2. (3X3) window and equation to encode the center pixel [6]

$3 \mathrm{X} 3$ window

$\mathrm{V} 1=\mathrm{B}_{2}+\mathrm{B}_{8}-2 \mathrm{~B}_{1}$

$\mathrm{V}_{\mathrm{i}}=\mathrm{B}_{\mathrm{i}+1}+\mathrm{B}_{\mathrm{i}-1}-2 \mathrm{~B}_{\mathrm{i}} \quad \mathrm{i}=1 \ldots \ldots 4$

$\mathrm{V}_{7}=\mathrm{B}_{8}+\mathrm{B}_{6}-2 \mathrm{~B}_{7}$

Where $I_{p}=V_{i}$ and $V_{i}$ will be calculated as in fig 3. Check $V_{i}$ values, if $V_{i}<0$, put it 0 ; otherwise put it 1. Finally, translate Vi values to decimal values by using V1 as the LSB and V4 as the MSB; $i=1$...... 4. Fig. 3 shows the calculation of DLBPC for each pixel.

The histogram was used to portray the spectrum's characteristics. The same process we have applied to database images and stored these features in a database. 


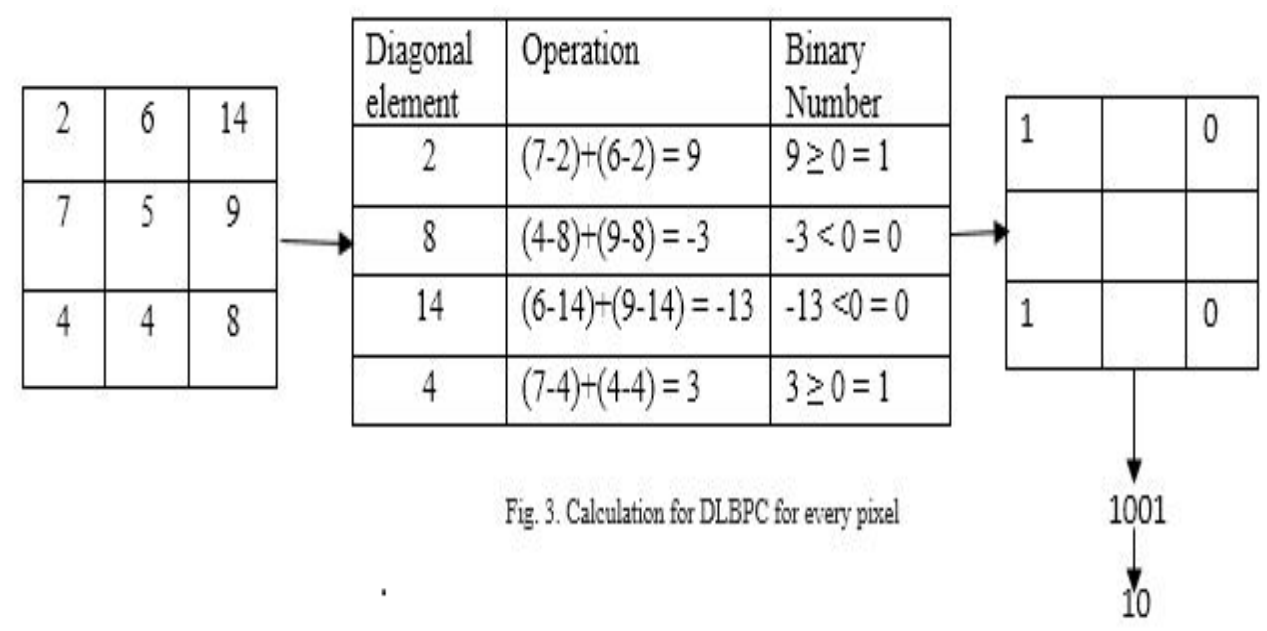

We represent the DLBPC as follows:

$$
\operatorname{LBP}\left(\boldsymbol{X}_{c}, \boldsymbol{y}_{c}\right)=\sum_{p=0}^{p-1} S\left(I_{p}\right) \times 2^{p}
$$

Step 3: Similarity matching \& Retrieval: This is the last step of every image retrieval system. After the step of extracting the feature of query images, similarity matching is performed between a query image and the respective repository images. The images are sorted in increasing order of the value of the distance metric used. Different types of distance metrics are there for the measurement of similarity, here in these experiments we have used City Block, Canberra, and Euclidean Distance and also proposed a new distance metric which is mentioned below. The experimental result shows that our proposed new distance metric outperforms better results than other distance metrics.

\subsection{Proposed (DLBPC + color moment) approach}

Step 1: Preprocessing: Same procedure has been applied as in section 2.2 of Step 1.

Step 2: Feature Extraction: This step is dependent on the avulsions of the image features by DLBPC and Color Moment that makes a combined feature vector. Our second approach boosts the discriminative power of the proposed (DLBPC) local color texture feature. In this, we have used L*a*b color space and extracted statistical values. These values include: Mean is the first, standard deviation is the second and skewness is the third statistical moment and calculated by the following equations:

$$
\begin{gathered}
\text { mean }=\frac{1}{M N} \sum_{i=1}^{N} \sum_{j=1}^{M}(I(i, j) \\
\text { std }=\sqrt{\frac{1}{M N}} \sum_{i=1}^{N} \sum_{j=1}^{M}(I(i, j)-\text { mean })^{2} \\
\text { skewnes }=\sqrt[3]{\frac{1}{M N}} \sum_{i=1}^{N} \sum_{j=1}^{M}\left(\frac{I(i, j)-\text { mean }}{s t d}\right)^{3}
\end{gathered}
$$

In all equations, I refer to one of the color channels of an input image, and M, N are its measures. For each channel of the $\mathrm{L}^{*} \mathrm{a} * \mathrm{~b} *$ color space, these three statistical parameters are determined. As a result, a total of 9 color features are produced, resulting in a feature vector for the color image.

After that, we combined the feature vector of the Diagonal local binary pattern for color image (DLBPC), and global features (color moment) of the image, and that feature vector length is 265 . The same process we have applied on database images and stored these features in the database.

Step 3: Similarity matching \& Retrieval: This is the same procedure that we have applied in Step 3 of section B. 


\section{Similarity Measures}

Searching for data items that are identical to a given query element using a similarity (or distance) feature is a common activity in many application domains, including multimedia knowledge retrieval, data processing, pattern recognition, and computational biology, to name a few. In the exact-match scenario, objects whose key match the query one. Therefore, exact-match has little meaning when dealing with complex data such as image, audio, video, except for limited applications like a search for complex and unstructured data. For example, in the case of CBIR, the search is not computed at the level of the actual images (e.g. pixel by pixel comparison) or as an exact match of the image representations, because even a small perturbation of an image may affect the numerical values of its pixel intensities and the associated image features.

The search is rather computed at the level of proximity of the image representations: the images whose representations are closest to the representation of the query are top-ranked. We'll use either a similarity function or a distance function to search a database for objects, much like a query. Within the first case, we look for the objects with the best similarity to the query. Within the latter case, we look for objects with the lowest distance from the query. If the ranked list of the responses to a query is the same, a similarity function is said to be identical to a distance function. There are many distance metrics like Euclidean, City Block, Canberra, Manhattan, BrayCurtis, Square chord, etc. This study compares the result of Euclidean, City Block, Canberra, and our proposed New Distance Metric.

\subsection{Proposed new Distance Metric}

We present a novel distance metric in this article. Assuming A and B are two attribute vectors from the query and repository images, respectively, with each variable $a_{i}$ and $b_{i}$, new distance metrics (NDM) are defined as:

$$
\operatorname{NDM}(A, B)=\sum_{i=1}^{n}\left|a_{i}^{1 / p}-b_{i}^{1 / p}\right|
$$

Where $\mathrm{p}$ is a positive integer parameter, $\mathrm{p} \in \mathrm{N}+$.

Where $\mathrm{p}=1$, the new distance metric is the same as city Block distance, so we confine in our approach $\mathrm{p}>1$. In our proposed approach we have used $\mathrm{p}=3$, that outperform better result than other distance metrics (like Euclidian, city block, Canberra). We have used a new distance metric with our proposed Diagonal local binary pattern for color images (DLBPC) which when combined color moment (DLBPC+ Color moment) results in an efficient image retrieval technique. We have used different types of databases for simulation: Wang Database (1000 images), GHIM-10K (10000 images). Retrieval time is also low in our proposed approach.

\section{Analysis and Result}

\subsection{Performance \& Measures}

The two most basic and popular measures are precision and recall. The precision is the percentage of returned objects that are significant to the query, and it shows how accurate an algorithm is at returning significant objects. The recall (or sensitivity) is the fraction of significant objects that are successfully fetched, so it gives a measure of how complete an algorithm is in returning relevant objects [7].

Every image in the repository acts as a query image in our tests. The accuracy $\mathrm{P}(\mathrm{N})$ and recall $\mathrm{R}(\mathrm{N})$ for fetching the top $\mathrm{N}$ items, as defined in [52,53], are used to evaluate an image retrieval system's efficiency.

$$
P(N)=\frac{l_{N}}{N} \quad ; \quad R(N)=\frac{I_{N}}{M}
$$

As above equation (10), $\mathrm{I}_{\mathrm{N}}$ denotes the number of comparable images gathered from the top $\mathrm{N}$ locations and $\mathrm{M}$ denotes the total number of comparable images in the repository. The average accuracy of a single query $\bar{p}(q)$ is the mean of all precision values $\mathrm{p}(\mathrm{n}), \mathrm{n}=1,2, \ldots \ldots \ldots \mathrm{N}$

$$
\bar{p}(q)=\frac{1}{N} \sum_{n-1}^{N} p(n)
$$


The mean average precision (mAP) is the average of the total query scores Q:

$$
m A P=\frac{1}{Q} \sum_{q=1}^{Q} \bar{p}(q)
$$

\subsection{Datasets}

Wang Database [55]: It comprises 1000 images divided into ten classes, each with 100 images. Each class includes images that are either $256 \times 384$ pixels or $384 \times 256$ pixels in size. African people, beach, house, bus, dinosaur, elephant, flower, horse, glacier, and food are among the 10 groups in the Wang $1 \mathrm{~K}$ database [55].

GHIM-10K Database [56]: The GHIM-10K dataset is the second one. All of the photographs in this dataset were gathered by the author from the web and a camera (Guang-Hai Liu). Fireworks, Western Architecture, Car, Motorcycle, Sailing Boat, Helicopter, Butterfly, The Great Wall, Dragonfly, Snowberg, Flower, Tree, Field, Beach, Sunset, Chinese Architecture, Chicken, Insect, Horse, and Aircraft Carrier are among the 10,000 photos used. Each type includes 500 JPEG images with a size of $400 \times 300$ or $300 \times 400$ pixels. The majorities of the original images are high-resolution and can be zoomed down to $400 \times 300$ or $300 \times 400$ pixels.

\subsection{Obtained Results}

This section contains a variety of experimental observations that show the feasibility of the suggested approaches and equate them to the effects of the closely related active structure LBP, LBPC, MSLBP, MDLBP, and LCVBP. We led the thorough image retrieval analysis and discovered that their design approach and filter parameters outperformed the other implementations. We used MATLAB-R2019B in a Microsoft Windows environment with an $\mathrm{I7}$ CPU and $8 \mathrm{~GB}$ of main memory to test our proposed approaches. Since the LBP operator is added to each part of the pixel, the LBP feature vector for a color image has a dimension of 768. MSLBP, MDLBP, LCVBP, DLBPC, DLBPC+ Color moment function vectors have dimensions of 2304, 2048, 236, 256, and 265 each. However, the feature vector length in all approaches (MSLBP and MDLBP) is the longest (2304, 2048), which would slow down the system's retrieval performance. The accuracy versus recall values for seven methods (2 proposed, 5 existing) that provide the best overall results are plotted in Fig.4. DLBPC and DLBPC + Color moment are the two proposed methods, while MDLBP, MSLBP, LCVBP, LBP, and LBPC are the five existing methods.

\begin{tabular}{|c|c|c|c|c|c|}
\hline \multirow[b]{2}{*}{ Method } & \multirow{2}{*}{$\begin{array}{l}\text { No. of } \\
\text { features }\end{array}$} & \multicolumn{4}{|c|}{ Distance Metric } \\
\hline & & Euclidean & City block & Canberra & $\begin{array}{c}\text { New Distance } \\
\text { metric } \\
\text { (Proposed) }\end{array}$ \\
\hline LBP [14] & $3 * 256=768$ & 55.28 & 55.23 & 56.93 & \\
\hline LBPC [7] & 256 & 55.49 & 55.05 & 58.05 & \\
\hline MSLBP[38] & 2304 & 59.86 & 59.83 & 60.62 & \\
\hline MDLBP[47] & 2048 & 59.58 & 59.58 & 60.82 & \\
\hline LCVBP $[50]$ & 236 & 53.42 & 53.44 & 56.83 & \\
\hline DLBPC(proposed) & 256 & 57.34 & 57.75 & 60.89 & 61.72 \\
\hline $\begin{array}{c}\text { DLBPC+ color } \\
\text { moment (proposed) }\end{array}$ & 265 & 58.59 & 58.98 & 64.61 & 68.61 \\
\hline
\end{tabular}

Table 1 For $\mathrm{N}=100$, mean average precision (mAP) is present as a result of different approaches on the Wang-1K dataset using various distance metrics and a New distance metric.

Table 1 shows the mAP values derived using different methods for the top one hundred images $(\mathrm{N}=100)$ on the Wang-1000 datasets. The top techniques are bolded within the order of their retrieval performance for ease. As query images, all database images are used. The suggested DLBPC and DLBPC + color moment using the 
suggested new distance metric receive the best mAP values of 61.72 and 68.61 , respectively, as shown in the table. In our suggested approach, the feature vector length is also low which will speed up the retrieval performance. DLBPC + color time has the third greatest mAP value of 64.61 when using Canberra distance. DLBPC has the next best mAP value of 60.89 when using the Canberra distance metric. MDLBP, using the Canberra distance metric, achieves the fifth-greatest mAP value of 60.82. MSLBP, using the Canberra distance metric, came in second with a mAP of 60.62 .

The graph (Fig 4) reveals that for all recall values, the suggested methods constantly have the fastest retrieval time, led by MDLBP, MSLBP, LBPC, LCVBP, and LBP.

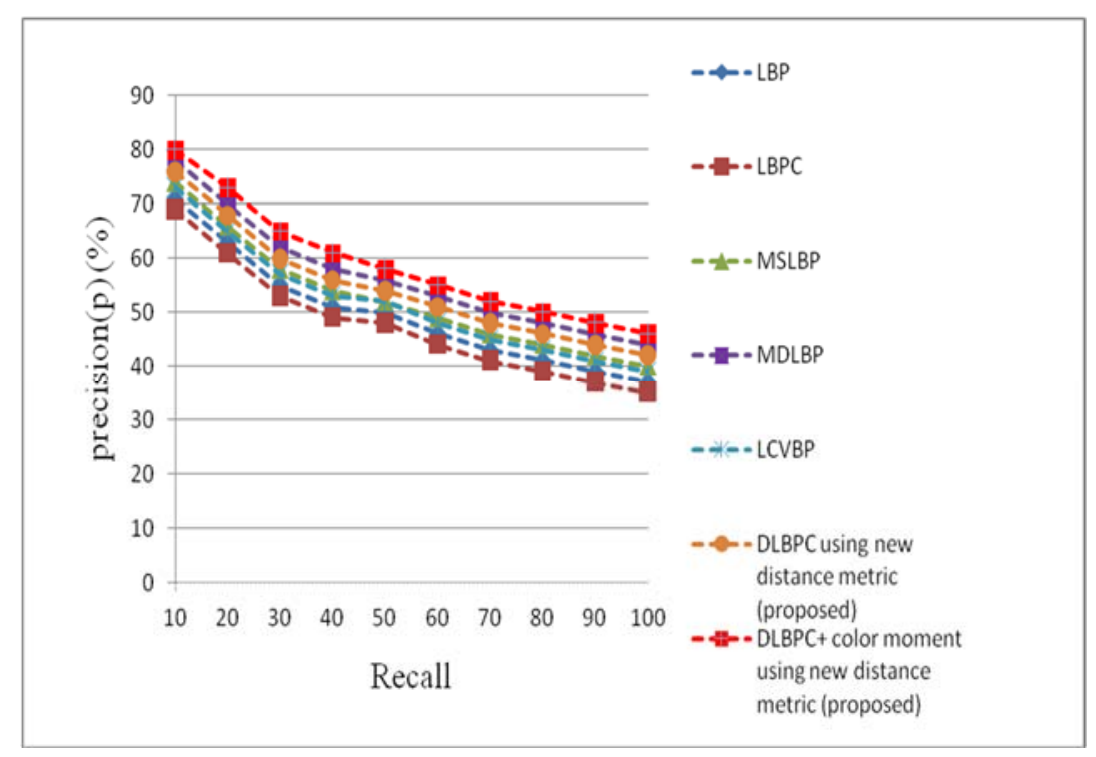

Fig. 4. P-R curves $n=1$ to 100 on the Wang dataset for the two suggested methods DLBPC, DLBPC + color moment, and five active methods

As seen in the above graph, the suggested approaches consistently have the highest retrieve rates for all recall values, followed by MDLBP, MSLBP, LCVBP, LBP, and LBPC. The consistency trend is the same with all recall values $\mathrm{N}=1$ to 100 .

\begin{tabular}{|c|c|c|c|}
\hline \multicolumn{1}{|l|}{ Class } & $\begin{array}{c}\text { DLBPC+Color moment using a } \\
\text { new distance metric (proposed) }\end{array}$ & $\begin{array}{c}\text { MDLBP [46 ] using } \\
\text { Canberra distance metric }\end{array}$ & Difference \\
\hline African people & 63.03 & 55.75 & 7.28 \\
\hline Beach & 46.12 & 45.21 & 0.91 \\
\hline Building & 68.20 & 56.81 & 11.39 \\
\hline Bus & 90.10 & 80.79 & 9.31 \\
\hline Dinosaur & 97.01 & 97.10 & -0.09 \\
\hline Elephant & 56.05 & 41.53 & 14.52 \\
\hline Flower & 96.12 & 80.08 & 16.04 \\
\hline Horse & 70.23 & 61.61 & 8.62 \\
\hline Mountain & 46.10 & 33.8 & 12.3 \\
\hline Food & 53.15 & 55.54 & -2.39 \\
\hline Average & 68.61 & 60.82 & 7.79 \\
\hline
\end{tabular}

Table 2 DLBPC+ Color moment using current distance metric and MDLBP [46] using Canberra distance metric on Wang-1K dataset to obtain class-wise mean average precision (mAP) in percent for $\mathrm{N}=100$

Table 2 shows the utility of the suggested (DLBPC + Color moment) and real approaches (MDLBP). The flower class mAP yields $96.12 \%$ in our suggested approach, which is substantially higher than MDLBP's yields of 80.08 percent. Elephant, mountain, house, bus, and horse all have notable differences, with 14.52, 12.30, 11.39, 9.31, 
and 8.62, respectively. For each value of $\mathrm{N}(\mathrm{N}=1$ to 10$)$ on the Wang database, the number of valid images obtained by MDLBP (Method R) and the suggested method DLBPC+ Color moment (Method S). Having these variations our proposed methods give better retrieval results than MDLBP. Horses, mountains, buildings, buses, and African people are among the other classes for which our proposed methods produce considerably improved results. These classes, like the elephant, come in a multitude of shapes and sizes. Other courses don't have as many picture variants. A more detailed analysis of the number of relevant results was obtained.

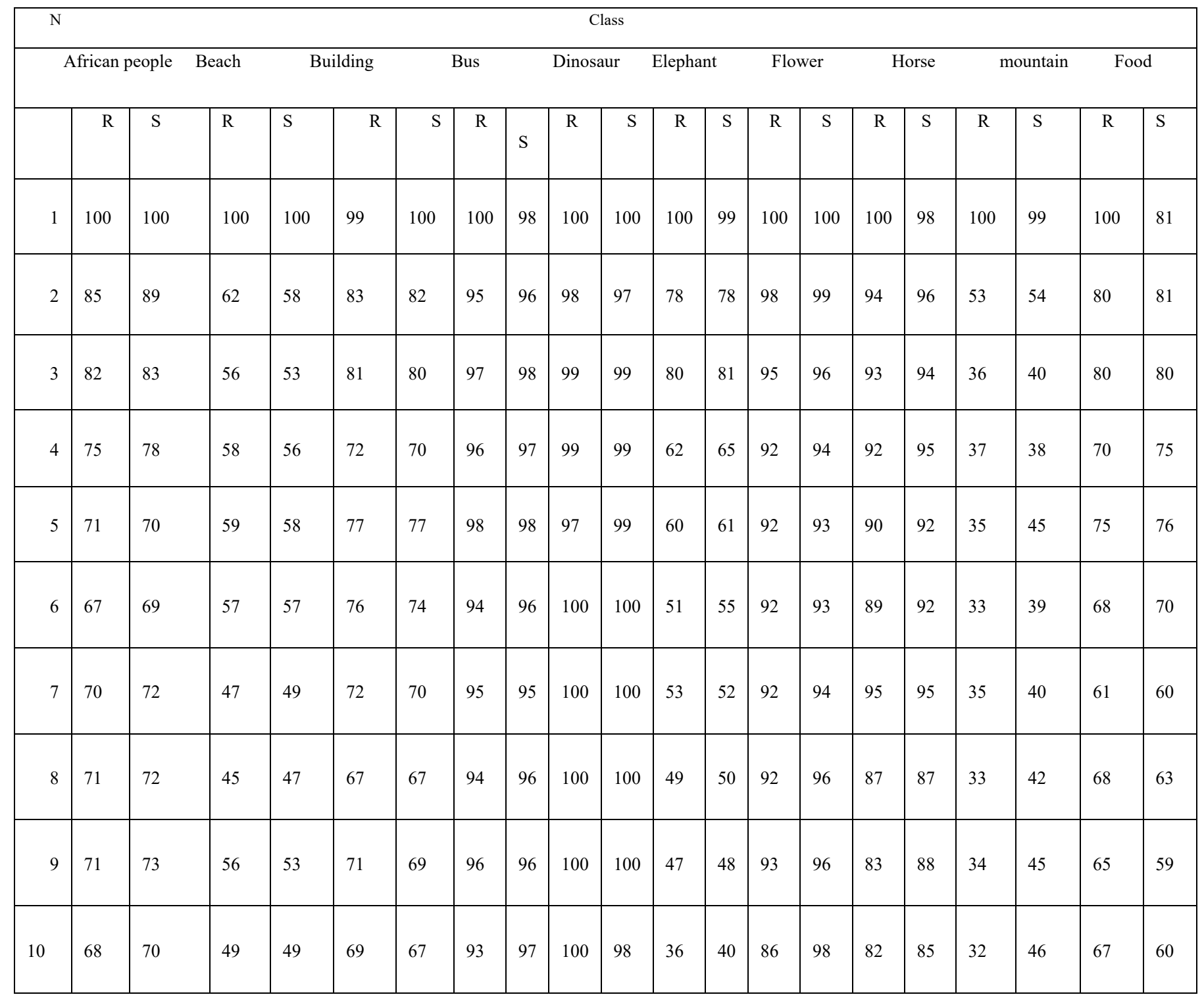

Table 3: For each value of $\mathrm{N}(\mathrm{N}=1$ to10) on the Wang database, the number of valid images obtained by MDLBP ( $\mathrm{R})$ and the suggested method DLBPC+ Color moment (S).

For each of the classes, the table shows the number of related images obtained by MDLBP (R) and DLBPC+ Color moment (S) at position $\mathrm{N}=1,2 \ldots \ldots .10 . \mathrm{N}$ and the result for $\mathrm{N}=1$ to 10 are presented in Table 3 out of 100 . 


\begin{tabular}{|c|c|c|c|c|c|}
\hline \multirow[b]{2}{*}{ Method } & \multirow{2}{*}{$\begin{array}{l}\text { No. of } \\
\text { features }\end{array}$} & \multicolumn{4}{|c|}{ Distance Metric } \\
\hline & & Euclidean & City Block & Canberra & $\begin{array}{c}\text { New Distance } \\
\text { metric(proposed) }\end{array}$ \\
\hline LBP [14] & $\begin{array}{c}3 * 256=7 \\
68\end{array}$ & 28.97 & 29.98 & 29.33 & \\
\hline LBPC [7] & 256 & 24.45 & 25.89 & 27.25 & \\
\hline MSLBP [38] & 2304 & 28.99 & 28.86 & 31.69 & \\
\hline MDLBP [47] & 2048 & 31.83 & 33.23 & 33.97 & \\
\hline LCVBP[50] & 236 & 29.25 & 29.26 & 29.72 & \\
\hline $\begin{array}{c}\text { DLBPC using } \\
\text { new distance } \\
\text { metric } \\
\text { (proposed) }\end{array}$ & 256 & 27.86 & 28.46 & 33.99 & 40.23 \\
\hline $\begin{array}{c}\text { DLBPC+ } \\
\text { color moment } \\
\text { using new } \\
\text { distance } \\
\text { metric } \\
\text { (proposed) } \\
\end{array}$ & 265 & 31.63 & 31.65 & 34.84 & 42.12 \\
\hline
\end{tabular}

Table 4. For $\mathrm{N}=100$, the mean average precision (mAP) was found using different approaches on the GHIM-10K dataset using various distance metrics and the New distance metric.

The number of relevant images obtained by Method S for the category Horse is substantially higher than those obtained by Method R, except for N=1, where Method R adds two more relevant images than Method S.

For each value of $\mathrm{N}$, images were created to match the performance and the results for $\mathrm{N}=1$ to 10 is shown in Table 3. For each of the classes, the table shows how many related images were obtained by MDLBP (Method R) and DLBPC+ Color moment (Method $\mathrm{S}$ ) at position $\mathrm{N}=1,2 \ldots \ldots .10$. With the case of $\mathrm{N}=1$, where Method $\mathrm{R}$ generates two more relevant images than Method $\mathrm{S}$, the number of relevant images produced by Method $\mathrm{S}$ for the group Horse is slightly greater than that obtained by Method R.

Table 4 contains the recovery findings for GHIM-10K. The mAP values have a similar tendency to Wang datasets. The highest mAP values are 42.12 percent and 40.23 percent, respectively, for the suggested methods $\mathrm{DLBPC}+$ Color moment and DLBPC using the proposed new distance metric. DLBPC+ Color time, using the Canberra distance metric achieves the third-largest value of mAP, which is 34.84 percent. DLBPC, using the Canberra distance metric, achieves the next best mAP value of 33.99. MDLBP comes in second with a value of 33.97 percent, and MSLBP comes in fifth with a value of Images were created to match the performance for each value of 31.69 .

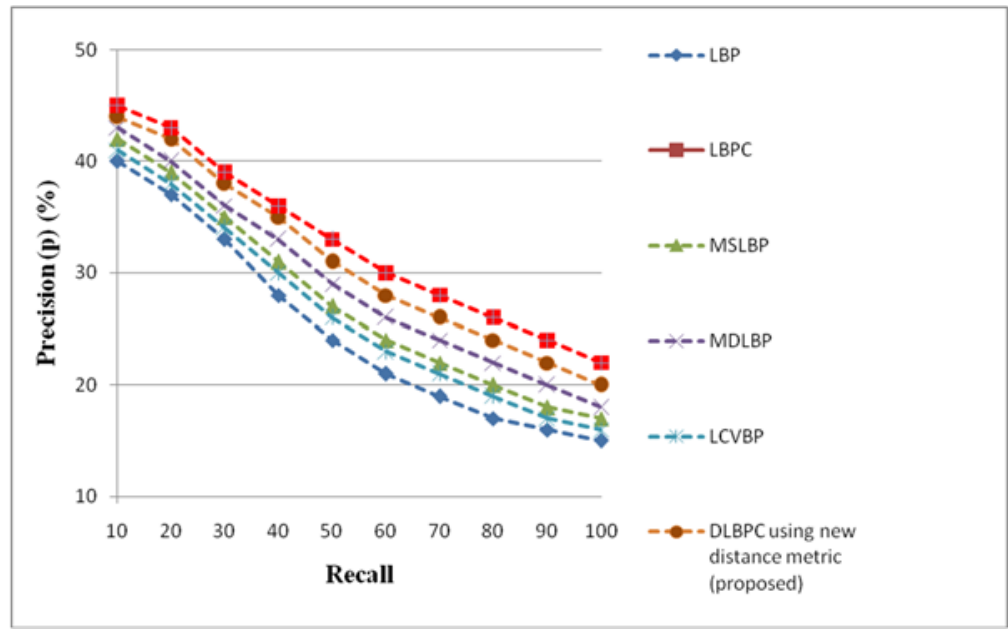

Fig. 5. P-R curves for the five active methods MDLBP, MSLBP, LCVP, LBPC, LBP, and two proposed methods DLBPC + color moment and DLBPC for $\mathrm{N}=1$ to 100 on GHIM-10K. 
The accuracy versus recall values of GHIM-10K for $\mathrm{N}=1$ to 100 is shown in Figure 5. For all recall values, the proposed techniques use greater accuracy. The mAP value for all methods decreases as the data set is larger.

\subsection{Time Analysis}

The three time-consuming procedures are feature extraction, distance calculation $\mathrm{D}(\mathrm{P}, \mathrm{Q})$ for a given query $\mathrm{p}$ and repository image $\mathrm{q}$, and sorting of the top $\mathrm{N}$ image from a repository of $\mathrm{R}$ images. The size $\mathrm{L}$ of the attribute vector affects the calculation of $\mathrm{D}(\mathrm{P}, \mathrm{Q})$. This move has an order of time complexity of $\mathrm{O}(\mathrm{L})$. To recover $\mathrm{N}$ top images from $\mathrm{R}$ repository images, we employ a basic sorting algorithm (bubble sort) with time complexity of $\mathrm{O}$ $(\mathrm{N}, \mathrm{R})$. This is true for all of the procedures. We called it retrieval time because we combined the times for distance. Table 5 shows the time for property extraction, retrieval time, and cumulative time for all seven processes. The dimension of the function vector is also included in the table. With $0.051(\mathrm{~s})$ and $0.967(\mathrm{~s})$ for attribute extraction and image retrieval, LCVBP is the fastest. The property extraction and retrieval times for our proposed systems DLBPC and DLBPC+Color moment are 0.037(s), 0.0972(s) and 0.039(s), 0.981(s) respectively.

\begin{tabular}{|c|c|c|c|c|c|}
\hline Sr. no & Methods & No. of features & $\begin{array}{l}\text { Property } \\
\text { Extraction } \\
\text { Time (s) }\end{array}$ & $\begin{array}{l}\text { Retrieval } \\
\text { Time(GHIM- } \\
10 \text { dataset) }\end{array}$ & $\begin{array}{l}\text { Total } \\
\text { Time(s) }\end{array}$ \\
\hline 1 & LBP [14] & $3 * 256=768$ & 0.099 & 1.885 & 1.984 \\
\hline 2 & LBPC [7] & 256 & 0.038 & 0.977 & 1.015 \\
\hline 3 & MSLBP [38] & 2304 & 0.731 & 4.074 & 4.805 \\
\hline 4 & MDLBP [47] & 2048 & 0.212 & 2.126 & 2.428 \\
\hline 5 & $\begin{array}{c}\text { LCVBP [50] } \\
6\end{array}$ & 236 & 0.051 & 0.967 & 1.018 \\
\hline 7 & $\begin{array}{c}\text { DLBPC(usin } \\
\text { mew distance } \\
\text { metric)Proposed }\end{array}$ & 256 & 0.037 & 0.972 & 1.009 \\
\hline & $\begin{array}{c}\text { DLBPC+ } \\
\text { color moment } \\
\text { (using new } \\
\text { distance } \\
\text { metric)Proposed }\end{array}$ & 265 & 0.039 & 0.981 & 1.020 \\
\hline
\end{tabular}

Table 5: Various methods require time (seconds) to extract features and retrieve images from the top 100 images in the GHIM- 10K datasets.

\section{Conclusion}

For data retrieval with high precision and time, DLBPC and a new distance metric are proposed. DLBPC with suggested distance metric uses $\mathrm{L}^{*} \mathrm{a}^{*} \mathrm{~b}^{*}$ color space and threshold of diagonal color pixel during circularly symmetric neighborhood of a pixel of evenly spaced member P and radius R. DLBPC was combined with Color moment for effective retrieval (global Feature). Across all datasets, the proposed approach DLBPC + Color moment using the proposed new distance metric achieves better value of mAP than the commonly used multichannel decoded local binary pattern (MDLBP). Further research let out the suggested descriptor is also useful when there is a lot of intra-class variance among the images.

The proposed method was implemented on daunting datasets: Wang-1K, GHIM-10K datasets to test the efficacy of our image retrieval system, and found competitive in terms of accuracy and speed over the other methods.

\section{Acknowledgement}

This paper and analysis behind it would not have been possible without the excellent guidance of my guide, Dr. Prof. Rajesh Ranjan His enthusiasm, experience, and careful attention to detail have motivated me to complete this study.

\section{References}

[1] Y. Rui, T. Huang and S. Chang, "Image Retrieval: CurrentTechniques, Promising Directions, and Open Issues,"Journal of Visual Communication and Image Representation, vol. 10, no.1, pp. 39-62, 1999.

[2] A. Smeulders, M. Worring, S. Santini, A. Gupta and R. Jain,"Content-based image retrieval at the end of the early years,"IEEE Transactions on Pattern Analysis and Machine Intelligence, vol. 22, no. 12, pp. 1349-1380, 2000.

[3] M. Kokare, B. Chatterji and P. Biswas, "A Survey on Current Content based Image Retrieval Methods,"IETE Journal of Research, vol. 48, no. 3-4, pp. 261-271, 2002. 
[4] Y. Liu, D. Zhang, G. Lu and W. Ma, "A survey of contentbased image retrieval with high-level semantics," Pattern Recognition, vol. 40, no. 1, pp. 262-282, 2007.

[5] R. Datta, D. Joshi, J. Li and J. Wang, "Image retrieval," ACM Computing Surveys, vol. 40, no. 2, pp. 1-60, 2008.

[6] M. Alrahhal, K. P. Supreethi, "Content-Based Image Retrieval using Local Patterns and Supervised Machine Learning Techniques," In Proc. IEEE International Conference on Artificial Intelligence (AICAI)' 2019, pp. 118-124

[7] C. Singh, E. Walia, K.P Kaur, "Color texture description with novel local binary patterns for effective image retrieval," Pattern Recognition, vol. 76, no.2, pp. 50-68, 2018

[8] B.S. Manjunath , J. R. Ohm, V.V. Vasudevan and A. Yamada , "Color and texture descriptor," IEEE Transactions on Circuits and Systems for Video Technology, vol. 11, no. 6, pp. 703-715, 2001.

[9] H. Tamura, S. Mori and T. Yamawaki " "Texture features corresponding to visual perception," in IEEE Transaction System, Man,and Cybernetics, vol. 8, no.6, pp. 460-473, 1978

[10] B.S. Manjunath and W.Y. Ma, "Texture features for browsing and retrieval of image data," in IEEE Transaction Pattern Analysis and Machine Intelligence, vol. 18, no. 8, pp. 837-842, 1996.

[11] R.M. Haralick and K. Shangmugam, "Textural feature for image classification," in IEEE Transaction System, Man and Cybernetics, vol. 3, no.6, pp. 6610-621,1973.

[12] G. Cross , A. Jain, "Markov random field texture models," in IEEE Transaction Pattern Analysis and Machine Intelligence, vol. 5, no. 1, pp. 25-39, 1983.

[13] D.G. Lowe,"Distinctive image features from scale-invariant keypoints," International Journal of Computer Vision,vol. 60, No.2, pp.91110,2004

[14] T. Ojala , M. Pietikäinen and T. Mäenpää, "Multiresolution gray-scale and rotation invariant texture classification with local binary patterns," IEEE Transaction Pattern Analysis and Machine Intelligence, vol. 24 , no.7, pp. 971-987, 2002

[15] H. Bay, A. Ess, T. Tuytelaars, L.J.V. Gool , "SURF: speeded-up robust features," Computer Vision Image Understand., vol.110, no. 3,346-359, 2008 .

[16] E. Tola , V. Lepetit, P. Fua, "DAISY: an efficient dense descriptor applied to wide baseline stereo," IEEE Transaction Pattern Analysis and Machine Intelligence, vol. 32, no. 5, pp.815-830,2010 .

[17] Y. Ke , R. Sukthankar, "PCA-SIFT: a more distinctive representation for local image descriptors," In Proc. IEEE Conference on Computer Vision and Pattern Recognition’ 2004, pp. 506-513.

[18] N. Dalal , B. Triggs , " Histograms of oriented gradients for human detection," In Proc. IEEE Conference on Computer Vision and Pattern Recognition’ 2005, pp. 886-893.

[19] M. Calonder, V. Lepetit, M.O. Zuysal, T. Trzcinski , C. Strecha and P. Fua, “ Brief: com- puting a local binary descriptor very fast,” IEEE Transaction Pattern Analysis Machine Intelligence, vol.34, no. 7, pp.1281-1298, 2012 .

[20] J. Chen, S. Shan , C. He, G. Zhao , M. Pietikäinen, X. Chen and W. Gao, “ WLD: a robust local image descriptor," IEEE Transaction Pattern Analysis Machine Intelligence, vol.32, no. 9, pp. 1705-1720, 2010 .

[21] B. Li , R. Xiao , Z. Li , Z. Cai , B-L. Lu and L. Zhang, "Rank-SIFT: learning to rank repeatable local interest points," In Proc. IEEE Conference on Computer Vision and Pattern Recognition' 2011, pp. 1737-1744

[22] J. Vogel and B. Schiele, "Performance evaluation and optimization for content-based image retrieval," Pattern Recognition,vol. 39. No. $5,897-909,2006$

[23] D. S. Zhang and G. Lu, "Review of shape representation and description techniques," Pattern Recognition, vol. 37, pp.1-19, 2004.

[24] L.J.V.Gool,M. Everingham, C.K.I. Williams, J. Winn and A. Zisserman, "The Pascal visual object classes challenge," International Journal Computer Vision, vol. 88, no. 7, pp.303-338, 2010 .

[25] T. Mäenpää, M. Pietikäinen and T. Ojala, "Texture classification by multi-predicate local binary pattern operators," In Proc. International Conference on Pattern Recognition' 2000, pp. 3951-3954.

[26] T. Mäenpää, T. Ojala , M. Pietikäinen and M. Soriano, "Robust texture classification by subsets of local binary patterns," in Proc. International Conference on Pattern Recognition, 2000, pp. 3947-3950 .

[27] M. Pietikäinen, T. Mäenpää and V. Jaakko ,"Color texture classification with color histograms and local binary patterns," in: Workshop on Texture Analysis in Machine Vision, 2002, pp. 109-112 .

[28] Y. Guo , Z. Guoying and M. PietikäInen, "Discriminative features for texture description," Pattern Recognition, vol. 45, no. 6, 3834$3843,2012$.

[29] T. Ahonen, A. Hadid, M. Pietikäinen, "Face recognition with local binary pat terns," In proc. European Conference on Computer Vision' 2004, pp. 469-481.

[30] T. Ahonen, A. Hadid and M. Pietikäinen, "Face description with local binary patterns: application to face recognition,"IEEE Trans. Pattern Analysis Machine Intellegence, vol. 28 , no.12, pp. 2037-2041, 2006.

[31] C. Shan, S. Gong and P.W. McOwan, "Robust facial expression recognition using local binary patterns," in Proc. IEEE International Conference of Image Processing' 2005, pp. 370-373.

[32] C. Shan, S. Gong and P.W. McOwan, "Facial expression recognition based on local binary patterns: a comprehensive study," Image Vision Computuing , vol.27, pp.803-816, 2009

[33] S. Zhang, X. Zhao and B. Lei, "Facial expression recognition based on local binarypatterns and local fisher discriminant analysis," WSEAS Transaction Signal Processing, vol. 8, no.1, pp.21-31, 2012.

[34] Z. Guoying and M. Pietikainen, "Dynamic texture recognition using local binary patterns with an application to facial expressions," In IEEE Trans. Pattern Analysis' vol. 29, pp. 915-928, 2007.

[35] V.Sajwan and P.Goyal, "Sub-block features based image retrieval," Computational Intelligence in Data Mining, vol. 31, pp. 637-646, 2014.

[36] V. Takala, T. Ahonen and M. Pietikäinen, "Block-based methods for image retrieval using local binary patterns," in Proc. Scandinavian Conference on Image Analysis' 2005, pp. 882-891 .

[37] O.A.B. Penatti , E. Valle , R.S. Torres, "Comparative study of global color and texture descriptors for web image retrieval," Journal Vision Communication and Image Representation, vol. 23, no. 2, pp.359-380, 2012.

[38] T. Mäenpää, M. Pietikäinen, J. Viertola, "Separating color and pattern informa- tion for color texture discrimination," in Proc. 16th International Con- ference on Pattern Recognition' vol1, 2002, pp. 668-671 .

[39] T. Mäenpää and M. Pietikäinen, "Classification with color and texture: jointly or separately?," In Proc. Pattern Recognition' 2004, pp. $1629-1640$.

[40] C. Shan and T. Gritti, "Learning discriminative LBP-histogram bins for facial expression recognition," in Proc. British Machine Vision Conference (BMVC), 2008.

[41] R. Zabih and J. Woodfill, "Non-parametric local transforms for computing visual correspondence," In Proc. Euro. Conf. Computer Vision' 1994, pp. 151-158.

[42] Baochang Zhang, Yongsheng Gao, Sanqiang Zhao and Jianzhuang Liu, "Local Derivative Pattern Versus Local BinaryPattern:Face 
Recognition With High-Order Local PatternDescriptor," IEEE Transactions on Image Processing, vol. 19, no. 2, pp. 533-544, 2010.

[43] P. Reddy and K. Satya Prasad, "Local Derivative Patterns and their Magnitudes for Content Based Image Retrieval", International Journal of Computer Science \& Informatics, vol.1, no. 2, pp. 62-67, 2011.

[44] S. Murala, R. Maheshwari and R. Balasubramanian, "Local Tetra Patterns: A New Feature Descriptor for Content-Based Image Retrieval", IEEE Transactions on Image Processing, vol. 21, no. 5, pp. 2874-2886, 2012.

[45] M. Rahman, S. Antani and G. Thoma, "A Learning-Based Similarity Fusion and Filtering Approach for Biomedical Image Retrieval Using SVM Classification and Relevance Feedback," IEEE Transactions on Information Technology in Biomedicine, vol. 15, no. 4, pp. 640-646, 2011

[46] M. Nazari and E. Fatemizadeh, "A CBIR System for Human Brain Magnetic Resonance Image Indexing" ,International Journal of Computer Applications, vol. 7, no. 14, pp. 33-37,2010.

[47] S.R. Dubey, S.K. Singh and R.K. Singh, "Multichannel decoded local binary patterns for content-based image retrieval," IEEE Transactions in Image Processing, vol.25, no. 9, pp.4018-4032, 2016.

[48] J.Y. Choi , K.N. Plataniotis, Y.M. Ro ,Using local binary pattern features for face recognition, In Proc. 17th IEEE International Conference on Image Processing' 2010, pp. 4541-4544 .

[49] R. Lan , Z. Yicong and Y.T. Yuan, "Quaternionic local ranking binary pattern: a local descriptor of color images," IEEE Transaction Image Processing, vol. 25, no. 2, pp.566-579, 2015.

[50] J. Li , N. Sang and C. Gao, "Completed local similarity pattern for color image recognition," Neuro computing, vol.182, pp.111-117, 2016 .

[51] G-H Liu , J-Y Yang, “Content-based image retrieval using color difference histogram,” Pattern Recognition, vol. 46, pp.188-198, 2013.

[52] H. Abrishami Moghaddam, T Taghizadeh Khajoie, A.H. Rouhi and M. Saadatmand Tarzjan, "Wavelet Correlogram: A new approach for image indexing and retrieval," Pattern Recognition, vol. 38, pp. 2506 - 2518, 2005.

[53] H. Salehian,F.Jamzad and M. Jamzad, "Fast content Based Color Image Retrieval System Based on Texture Analysis of Edge Map," Advance Materials Research,vol.341, pp. 168-172, 2011.

[54] T. Mohana, V.Lalitha, L. Kusuma, N. Rahul and M. Mohana, "Various Distance Metric Methods for Query Based Image Retrieval," International Journal of Engineering Science and Computing, vol. 7, pp. 5818-5821, 2017.

[55] Wang database [online]. Available: http://www.wang .ist.psu.edu/docs/relate/

[56] GHIM-10K database [online] Available: http://www.ci.gxnu.edu.cn/cbir/Dataset.aspx.

\section{Authors Profile}
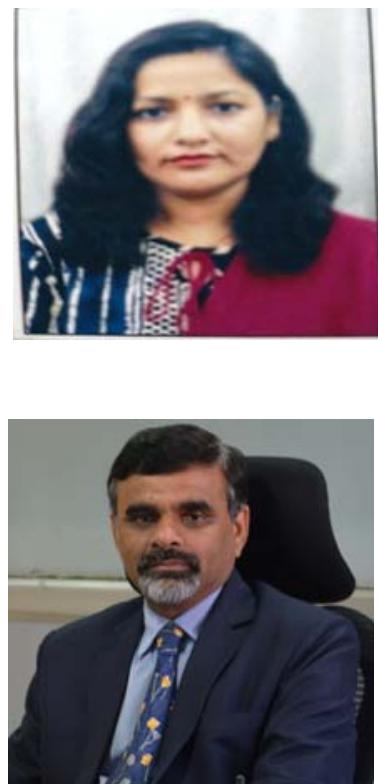

Prof.(Dr.) Rakesh Ranjan currently working as Vice Chancellor in Himgiri Zee University. He has done B.Sc(Engg) in Electrical Engineering from MIT Muzzaffarpur, Bihar University, ME from BIT Mesra and Ph.D. from BITS Pilani. Has guided 9 Ph.D students, 25 M.Tech thesis. He has published 79 International Research Paper, 06 text book. He got Siksha Rattan-2013. Educationist of the year at $7^{\text {th }}$ India Excellence Award, and Academy Award for Academic Excellence in Engineering Education.

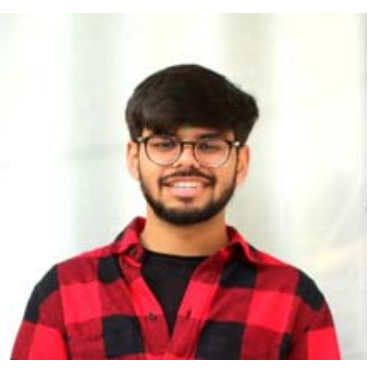

Ms. Vijaylakshmi Sajwan is currently working as Assistant Professor in Uttaranchal Institute of Management, Uttaranchal University. She has done BCA from C.C.S university, MCA from IGNOU, New Delhi and M-Tech in IT from Graphic Era University, Dehradun. Currently, pursuing Ph.D from Himgiri Zee University, Dehradun. Field of specialization- Image Processing. Has attended Seminars and Conferences at various levels, three paper published in international journals (including SPRINGER.

Sajal Swapnil is a final year B.Tech Student at NIT Kurukshetra in the department of Electronics and Communication Engineering. His area of interest lies with application of AI tools in Communication and has good knowledge of Data Structures and Algorithms using $\mathrm{C} / \mathrm{C}++$ and Python. He was Intern on roll at United Airlines Pvt Ltd for 6 months. Worked on Data Analysis and Visualization software like Spotfire and Tableau. Data modeling and Data Manipulation using SQL Server and Teradata. 